\title{
30. Biologie du vieillissement
}

\author{
(C) Springer-Verlag France 2010
}

\author{
30.1 \\ Altérations du protéome et vieillissement cellulaire \\ B. Friguet \\ Laboratoire de biologie cellulaire du vieillissement, UR4, IFR83, \\ université Pierre-et-Marie-Curie, Paris, France
}

L'augmentation de la quantité de protéines oxydées représente une caractéristique du vieillissement cellulaire. Cette accumulation de protéines modifiées avec l'âge résulte en partie d'une augmentation des espèces réactives de l'oxygène et d'autres composés toxiques provenant du métabolisme cellulaire et de facteurs environnementaux, mais de nombreux arguments expérimentaux indiquent qu'une défaillance de la maintenance des protéines (dégradation et réparation) représenterait une cause majeure de l'accumulation de ces protéines modifiées avec l'âge. La dégradation des protéines oxydées est principalement assurée par le protéasome dans le cytosol et le noyau tandis que la protease Lon a été impliquée dans la dégradation des protéines mitochondriales. Comme le protéasome représente le système majeur pour l'élimination des protéines endommagées et le renouvellement de protéines cytosoliques et nucléaires, son statut au cours du vieillissement a été l'objet de nombreuses études qui vont dans le sens d'une altération de la fonction protéasomale avec l'âge. La réparation des protéines oxydées ne concerne qu'un nombre limité de modifications oxydatives telle que l'oxydation du résidu méthionine qui peut être réversé grâce au système des méthionine sulfoxyde réductases (Msr) composé des enzymes MsrA et MsrB. Ces enzymes ont été impliqué dans la longévité et/ou la résistance contre le stress oxydant dans différents organismes ou modèles cellulaires. Ainsi, la methionine sulfoxyde reductase B2 (MsrB2) mitochondriale a été surexprimée de manière stable dans des cellules lymphoides MOLT-4. Suite à un traitement par $\mathrm{H}_{2} \mathrm{O}_{2}$, les cellules surexprimant MsrB2 présentent une viabilité accrue, une diminution de la quantité d'espèces réactives de l'oxygène et une fonction protéasomale préservée en comparaison avec des cellules témoins (Cabreiro et al (2008) J Biol Chem 283:16673-81). De plus, la surexpression de MsrA ou de MsrB2 dans les cellules MOLT-4 protège des effets pro-oxidants induits par le zinc et permet de prévenir les dommages oxydatifs des protéines (Cabreiro et al (2009) Antioxid Redox Signal 11:215-25). Par ailleurs, en utilisant la senescence réplicative des fibroblastes humains WI-38 comme modèle d'étude du vieillissement cellulaire, nous avons montré que les protéines oxydées et aussi les protéines modifiées par le produit de peroxydation lipidique 4-hydroxy-2-nonenal (HNE) et par glycoxidation (AGEs) étaient augmentées dans les fibroblastes WI-38 sénescents. L'identification des protéines sélectivement ciblées par l'oxydation et par les modifications avec le HNE et les AGEs, a montré qu'une majorité des protéines modifiées étaient d'origine mitochondriale, reflétant ainsi une accumulation préférentielle de protéines endommagées dans cette organelle au cours de la sénescence cellulaire (Ahmed et al (2010) Aging Cell 9:252-72).

\section{4}

Axe somatotrope et vieillissement : que nous apprennent les modèles animaux ? P. Gaudreau ${ }^{1}$, K. Bédard ${ }^{2}$

${ }^{1}$ Médecine

${ }^{2}$ Centre de recherche du CHUM, université de Montréal, Montréal, Canada

Introduction : Chez les mammifères, le vieillissement est caractérisé par une diminution de la sécrétion d'hormone de croissance $(\mathrm{GH})$, liée à une perte de sensibilité des cellules somatotropes au facteur de libération de la GH (GHRH). Chez le rat âgé, des modifications de niveaux d'ARNm du récepteur du GHRH (GHRH-R) ainsi qu'une diminution importante d'affinité de liaison du GHRH ont été rapportées. L'étude comparative de modèles animaux de vieillissement réussi, tel que le rat Sprague Dawley (SD) soumis à une restriction calorique modérée de longue durée (RC) et le rat Wistar/Lou nourri ad libitum (AL), offre une voie prometteuse dans le but de mieux comprendre les mécanismes biologiques menant à la dysfonction de cet axe neuroendocrinien au cours du vieillissement ou assurant sa protection.

Objectifs : Dans un premier temps, l'étude avait pour but de déterminer l'effet de la RC sur la somatotrope vieillissante, chez le rat SD, en ciblant principalement le GHRH-R comme molécule clé et d'identifier les mécanismes qui entrent en jeu pour maintenir sa fonctionnalité. Dans un second temps, l'étude visait à comparer les mécanismes de retardement de la somatopause dans les deux modèles animaux utilisés.

Méthodes : Des rats SD mâles de 18-20 mois, soumis à une RC à partir de l'âge de huit mois, ont été comparés à des rats âgés et de deux mois nourris AL. Les niveaux d'ARNm du GHRH-R et sa fonctionnalité (paramètres de liaison du GHRH, production d'AMPc, synthèse/ réparation d'ADN) ont été étudiés. De plus, une étude de régulation génique a été réalisée dans l'hypophyse antérieure des rats jeunes $\mathrm{AL}$ et âgés AL et RC ainsi que des mesures de concentrations de plusieurs marqueurs sériques. Une étude comparative a été menée chez des rats Lou mâles et femelles de différents groupes d'âge.

Résultats : Une augmentation des niveaux d'ARNm du GHRH-R hypophysaire a été observée chez les rats soumis à une RC contrairement à ceux de 2 et 18 mois AL. Chez les rats âgés RC, les études in vitro ont mis en évidence que les paramètres de liaison du GHRH, la production maximale d'AMPc stimulée par le GHRH et la synthèse/réparation d'ADN, stimulée par le GHRH, en condition hyperglycémiante, sont demeurés similaires à ceux des rats jeunes AL. Parmi les paramètres hormonaux et métaboliques étudiés affectant la fonction somatotrope, l'augmentation des niveaux sériques de glucocorticoïdes et la diminution des niveaux d'acides gras libres et de glucose par la RC pourraient contribuer au maintien de la fonctionnalité du GHRH-R. Des résultats similaires aux rats âgés RC ont été obtenus chez le rat Lou. Finalement, les nveaux d'ARNm de plusieurs gènes impliqués dans la transcription, la gestion du stress oxydatif et la régulation de la fonction somatotrope ont été affectés par le vieillissement ou la RC.

Conclusion : Cette étude fournit des pistes permettant d'identifier de nouveaux mécanismes impliqués dans le retardement de la somatopause et d'envisager des stratégies nutritionnelles moins contraignantes que la RC. 